\title{
Efficacy of infliximab in extrapulmonary sarcoidosis: results from a randomised trial
}

\author{
M.A. Judson*, R.P. Baughman ${ }^{\#}$, U. Costabel ${ }^{\mp}$, S. Flavin ${ }^{+}$, K.H. Lo ${ }^{+}$, M.S. Kavuru ${ }^{\S}$, \\ M. Drent ${ }^{f}$ and the Centocor T48 Sarcoidosis investigators**
}

ABSTRACT: The aim of the present study was to investigate the efficacy of infliximab for the treatment of extrapulmonary sarcoidosis.

A prospective, randomised, double-blind, placebo-controlled trial was conducted, with infliximab at 3 and $5 \mathrm{mg} \cdot \mathrm{kg}^{-1}$ body weight administered over 24 weeks. Extrapulmonary organ severity was determined by a novel severity tool (extrapulmonary physician organ severity tool; ePOST) with an adjustment for the number of organs involved (ePOSTadj). In total, 138 patients enrolled in the trial of infliximab versus placebo for the treatment of chronic corticosteroiddependent pulmonary sarcoidosis.

The baseline severity of extrapulmonary organ involvement, as measured by ePOST, was similar across treatment groups. After 24 weeks of drug-therapy study, the change from baseline to week 24 in ePOST was greater for the combined infliximab group compared with the placebo group. After adjustment for the number of extrapulmonary organs involved, the improvement in ePOSTadj observed in the combined infliximab group was also greater than that observed in placebo-treated patients, after 24 weeks of therapy. The improvements in ePOST and ePOSTadj were not maintained during a subsequent 24-week washout period.

Infliximab may be beneficial compared with placebo in the treatment of extrapulmonary sarcoidosis in patients already receiving corticosteroids, as assessed by the severity tool described in the present study.

KEYWORDS: Extrathoracic, infliximab, sarcoidosis, severity, therapy

S arcoidosis is a multisystem granulomatous disease of unknown cause. The disease may remit spontaneously or with treatment. Sarcoidosis is chronic, or progressive, in $\sim 25 \%$ of patients. Such patients require long-term therapy to avoid progressive organ dysfunction. Corticosteroids are currently recommended as the drug of choice for sarcoidosis $[1,2]$. However, the cumulative toxicities of corticosteroids make their long-term use problematic. Efforts should be made to taper corticosteroids to the lowest effective dose [1].

Due to the side-effects associated with long-term use of corticosteroids, there is interest in alternative therapies for sarcoidosis. Other than corticosteroids, drugs that have been studied in sarcoidosis have included methotrexate [3], hydroxychloroquine [4], azathioprine [5] and cyclophosphamide [6, 7].

Recently, the scientific literature has reported efficacy of tumour necrosis factor (TNF)- $\alpha$ antagonists for the treatment of pulmonary and extrapulmonary sarcoidosis [8-14]. There is a sound rationale for this therapy, because TNF- $\alpha$ is released by macrophages in patients with sarcoidosis [15], and TNF- $\alpha$ is thought to be integrally involved in the development of the granulomatous inflammation [16]. Furthermore, sarcoidosis patients whose disease is refractory to treatment with corticosteroids tend to have high levels of TNF- $\alpha$ in bronchoalveolar lavage fluid [17]. Since patients with extrapulmonary sarcoidosis, such as those with chronic skin conditions, upper respiratory tract and neurological involvement, tend to be recalcitrant to corticosteroid therapy $[6,18,19]$, TNF- $\alpha$ antagonists may be especially useful in these patients.

Infliximab is a chimeric immunoglobulin $\mathrm{G}$ monoclonal antibody directed against TNF- $\alpha$ [20]. A randomised, double-blind, placebo-controlled trial was conducted to evaluate the efficacy of infliximab for chronic corticosteroid-dependent
AFFILIATIONS

*Division of Pulmonary and Critical Medicine, Medical University of South Carolina, Charleston, SC, \#University of Cincinnati Medical Center, Cincinnati,

${ }^{\S}$ Dept of Pulmonary and Critical Care Medicine, Cleveland Clinic Foundation, Cleveland, $\mathrm{OH}$, +Centocor, Inc., Malvern, PA, USA, "Dept of Pneumology and Allergology, Ruhrlandklinik, Medical Faculty, University of DuisburgEssen, Essen, Germany. ${ }^{f}$ Dept of Respiratory Medicine, Sarcoidosis Management Team, University Hospital Maastricht, Maastricht, The Netherlands. **For full details of the Centocor T48 Sarcoidosis investigators, please see the Acknowledgements section.

CORRESPONDENCE

M.A. Judson, Division of Pulmonary and Critical Care Medicine, 96 Jonathan Lucas Street, Charleston, SC 29425, USA. Fax: 18437920732 E-mail: judsonma@musc.edu

Received:

April 302007

Accepted after revision:

January 252008

\section{STATEMENT OF INTEREST}

Statements of interest for M.A. Judson, R.P. Baughman, U. Costabel, S. Flavin, K.H. Lo and M.S. Kavuru can be found at

www.erj.ersjournals.com/misc/ statements.shtml 
pulmonary sarcoidosis [21]. The primary end-point of the study was improvement in forced vital capacity (FVC) after 24 weeks of treatment [21]. The effect of infliximab on extrapulmonary sarcoidosis organ involvement was evaluated as a secondary end-point in the present study. An extrapulmonary physician organ severity tool (ePOST) was developed for the current evaluation and the results of sarcoidosis extrapulmonary organ involvement in the trial were reported.

\section{METHODS}

\section{Eligibility}

Eligible adult patients were required to have histologically proven sarcoidosis, diagnosed $\geqslant 1$ yr prior to screening, and evidence of parenchymal disease (stage II or III) on chest radiographs. Additional eligibility criteria included an FVC of $50-85 \%$ of the predicted value and a Medical Research Council dyspnoea score of at least grade 1 [22]. Patients were required to have been treated with $\geqslant 10 \mathrm{mg} \cdot$ day $^{-1}$ of prednisone equivalent or one or more immunosuppressants for $\geqslant 3$ months prior to screening. Doses of these medications were to have remained stable for $\geqslant 1$ month prior to study entry. During the study, background medication regimen and doses were to remain stable.

Patients were excluded from the study if they had a history or current evidence of latent or active tuberculosis, chronic or serious infections within 2 months of screening, malignancy, or congestive heart failure. Previous administration of infliximab or other TNF- $\alpha$ inhibitors within 3 months of screening also excluded patients from participation.

\section{Study design}

The present study was a phase 2, multicentre, double-blind, placebo-controlled study, in which patients were randomised in a 1:1:1 ratio to receive intravenous infusions of either placebo, infliximab at $3 \mathrm{mg} \cdot \mathrm{kg}^{-1}$ body weight or infliximab at $5 \mathrm{mg} \cdot \mathrm{kg}^{-1}$ body weight, at weeks $0,2,6,12,18$ and 24 , and were followed through to week 52 . The design was selected in order to simultaneously assess the efficacy of infliximab

\begin{tabular}{|c|c|}
\hline TABLE 1 & $\begin{array}{l}\text { Organs evaluated in the extrapulmonary } \\
\text { physician organ severity tool score }\end{array}$ \\
\hline \multicolumn{2}{|c|}{ Skin } \\
\hline \multicolumn{2}{|c|}{ Peripheral lymph nodes } \\
\hline \multicolumn{2}{|c|}{ Eyes } \\
\hline \multicolumn{2}{|l|}{ Liver } \\
\hline \multicolumn{2}{|l|}{ Spleen } \\
\hline \multicolumn{2}{|c|}{ Central nervous system } \\
\hline \multicolumn{2}{|c|}{ Peripheral nervous system } \\
\hline \multicolumn{2}{|c|}{ Parotid/salivary glands } \\
\hline \multicolumn{2}{|c|}{ Bone marrow } \\
\hline \multicolumn{2}{|l|}{ Ear } \\
\hline \multicolumn{2}{|l|}{ Nose } \\
\hline \multicolumn{2}{|l|}{ Throat } \\
\hline \multicolumn{2}{|l|}{ Cardiac } \\
\hline \multicolumn{2}{|l|}{ Renal } \\
\hline \multicolumn{2}{|l|}{ Bone/joint } \\
\hline \multicolumn{2}{|l|}{ Muscle } \\
\hline Gastrointestinal & \\
\hline
\end{tabular}

(placebo versus the combination of the $3 \mathrm{mg} \cdot \mathrm{kg}^{-1}$ and $5 \mathrm{mg} \cdot \mathrm{kg}^{-1}$ groups) and determine whether there was a dose-response relationship (placebo versus $3 \mathrm{mg} \cdot \mathrm{kg}^{-1}$ versus $5 \mathrm{mg} \cdot \mathrm{kg}^{-1}$ groups). Randomisation was carried out by an interactive voice recognition system (IVRS). Subject allocation to treatment groups was performed using an adaptive stratified design with the following strata: 1) investigational site; and 2) the presence or absence of disfiguring facial sarcoidosis skin lesions (lupus pernio), as determined by the investigator. The IVRS assigned randomised subjects to a specific treatment assignment that was known only to the unblinded pharmacist at the study site. Assignments were not carried out by block randomisation but were adjusted by a computer program to maintain overall balance, using the strata described previously. Enrolment was performed by study investigators and coordinators. The first subject gave consent for enrolment on September 30, 2003 and the last subject visit was on September 30, 2005. Infliximab was provided by Centocor, Inc. (Malvern, PA, USA) and infusions were administered over at least a $2-h$ period.

In total, 138 patients from 34 centres, 20 in the USA and 14 in Europe (details in the Acknowledgements section), were randomised between September 30, 2003 and August 31, 2004. Institutional Review Boards/Ethics Committees at the 34 participating sites approved the study and patients provided written informed consent before any protocol-specific procedures were performed.

\section{Efficacy and safety evaluations}

The primary end-point was the change from baseline in the FVC \% predicted, at week 24 . The results of this primary endpoint have been previously described [21].

The efficacy of infliximab in the treatment of extrapulmonary sarcoidosis was assessed as a secondary end-point in the present study. The definition of involvement of sarcoidosis in an extrapulmonary organ was based on a clinical decision of the principal investigator at each clinical centre. The principal investigators were all experienced in the clinical presentation and management of sarcoidosis. Sarcoidosis organ assessment was performed using an ePOST that was designed for the purpose of the present study. The ePOST examined the state of sarcoidosis extrapulmonary organ involvement in 17 extrapulmonary organs (table 1). At each visit, each of the 17 organs was evaluated by the study investigator. Investigators were instructed to use all clinical information available to them, including laboratory analyses and assessments by subspecialists, to assess each organ system. Each organ was scored on a

\section{TABLE 2 Severity assessment of each organ}

\begin{tabular}{lc} 
Score & Description \\
\hline 0 & Not affected \\
1 & Slight \\
2 & Mild \\
3 & Moderate \\
4 & Moderate to severe \\
5 & Severe \\
6 & Very severe \\
\hline
\end{tabular}




\section{TABLE 3 Patient baseline characteristics}

\begin{tabular}{|c|c|c|c|}
\hline Subjects $n$ & 45 & 93 & 138 \\
\hline Male & $26(57.8)$ & $52(55.9)$ & $78(56.5)$ \\
\hline \multicolumn{4}{|l|}{ Race } \\
\hline Caucasian & $29(64.4)$ & $64(68.8)$ & $93(67.4)$ \\
\hline Other & $0(0.0)$ & $2(2.2)$ & $2(1.4)$ \\
\hline Extrapulmonary involvement & $30(66.7)$ & $62(68.1)^{\#}$ & $92(67.6)$ \\
\hline Time since sarcoidosis histologically proven yrs & $7.0 \pm 6.2$ & $6.9 \pm 6.2$ & $6.9 \pm 6.2^{\bullet}$ \\
\hline FVC L & $2.86 \pm 0.77$ & $2.82 \pm 0.78$ & $2.83 \pm 0.78$ \\
\hline FVC \% pred & $68.8 \pm 11.1$ & $68.6 \pm 9.1$ & $68.7 \pm 9.7$ \\
\hline
\end{tabular}

scale from 0 (not affected) to 6 (very severely affected; table 2). Therefore, the ePOST score could range from $0(0 \times 17)$ to 102 $(6 \times 17)$. Since the ePOST score was a summation of all extrapulmonary organ involvement and was not weighted for specific organ involvement, major changes in one organ may have had their effect on the ePOST score diluted by the number of organs affected. To adjust for this potential confounding effect, an adjusted ePOST (ePOSTadj) score was calculated, which equalled the ePOST score divided by the number of extrapulmonary organs involved at any time during the study. Although it was a secondary end-point, the scoring system for each organ was established a priori. However, the ePOST was constructed post hoc in order to examine whether infliximab had an effect on extrapulmonary sarcoidosis.

In an attempt to determine whether the organs of the most important clinical impact were affected to a greater or lesser degree than the other organs, separate ePOST scores were calculated for "major organs" (cardiac, central nervous system, peripheral nervous system, liver, bone marrow, renal and eyes) and "minor organs" (bone/joint, muscle, skin, spleen, nose, peripheral lymph nodes). The remaining organs were not included because of the small number of patients who had involvement in those organs.

Safety assessments were performed throughout, until week 52. All adverse events that occurred between visits were reported. Infusion reactions were defined as any adverse event that occurred during or within $1 \mathrm{~h}$ of completing the study agent infusion.

\section{Statistical analyses}

For the primary end-point, the treatment effect was tested using ANCOVA. The details of analysis and results have been described previously [21] and are not included in the present study.

As a post hoc analysis, the ePOST score, which was the sum of the severity scores for all 17 extrapulmonary organs, was summarised by visit using descriptive statistics. No formal statistical comparison was made. As part of the descriptive statistics, nominal p-values were produced based on an
ANCOVA model, similar to that for the primary end-point, for change from baseline. Descriptive statistics and nominal p-values were also produced for ePOSTadj.

Individual organ involvements were also evaluated in addition to the aggregation (i.e. ePOST). Due to the potential skewing of the data, a nonparametric test, Wilcoxon rank-sum test, was used to perform between-treatment comparisons. Nominal $\mathrm{p}$-values are provided. Since the comparisons are for descriptive purposes, no adjustment was made for multiple comparisons.

\section{RESULTS}

\section{Baseline characteristics}

The baseline characteristics of the enrolled patients are shown in table 3. Patients had a mean \pm SD (range) age of $47.0 \pm 9.3$ (45-50) yrs, had a slight $(56.5 \%)$ male predominance, had a history of sarcoidosis for $6.9 \pm 6.2 \mathrm{yrs}$ and had a baseline FVC of $68.7 \pm 9.7 \%$ pred, and approximately one-third $(29.7 \%)$ were black. Approximately two-thirds (92 out of $136 ; 67.6 \%$ ) of patients had extrapulmonary sarcoidosis involvement. Two patients who were randomised did not receive any study drug and, thus, an ePOST was not performed. Both of these patients were randomised to the infliximab $3 \mathrm{mg} \cdot \mathrm{kg}^{-1}$ group. The baseline clinical characteristics were comparable between the placebo and the combined infliximab groups.

Table 4 shows the proportions of study patients with extrapulmonary organ involvement at baseline. Figure 1 shows the number of extrapulmonary organs involved with sarcoidosis at baseline, according to treatment group. At baseline, the treatment groups were similar with regard to number of organs involved. As required by the study protocol, all patients had at least one organ, the lungs, affected. However, 25\% (33 out of 136) of patients presented with two organs involved, and it was quite common (53 out of $136 ; \sim 40 \%$ ) to have three to six organs affected.

\section{Changes in organ involvement}

Changes in organ involvement from baseline to week 24, as assessed by ePOST scores, are presented in table 5. The infliximab groups had fewer organs involved with sarcoidosis 


\begin{tabular}{lc}
\hline TABLE 4 & Frequency of organ involvement at baseline \\
\hline Organ & Patients affected \\
\hline Lungs & $136(100)^{\#}$ \\
Peripheral lymph nodes & $50(37)$ \\
Skin & $36(26)$ \\
Bone/joint & $27(20)$ \\
Liver & $19(14)$ \\
Eyes & $19(14)$ \\
Muscle & $18(13)$ \\
Cardiac & $12(9)$ \\
Peripheral nervous system & $11(8)$ \\
Nose & $11(8)$ \\
Central nervous system & $9(7)$ \\
Spleen & $8(6)$ \\
Renal & $8(6)$ \\
Bone marrow & $5(4)$ \\
Throat & $3(2)$ \\
Parotid/salivary glands & $3(2)$ \\
Ear & $1(1)$ \\
Gastrointestinal & $1(1)$ \\
\hline &
\end{tabular}

Data are presented as $\mathrm{n}(\%) .{ }^{*}$ : two patients were randomised but did not receive study agent; thus, organ involvement was not assessed.

at week 24 compared with baseline, in 38\% (34 out of 89 ) of patients compared with only $16 \%$ (seven out of 44 ) of those receiving the placebo.

The mean ePOST values over time in the placebo and combined infliximab groups are shown in figure 2. Although the mean \pm SEM ePOST value at baseline was slightly higher in the placebo group than in the infliximab group $(4.00 \pm 0.81$ versus $3.55 \pm 0.43$, respectively), the difference was within variability. The mean \pm SEM ePOST values at week 24 for the combined infliximab and placebo groups were $2.09 \pm 0.32$ and $3.70 \pm 0.85$, respectively. The improvement in ePOST scores at week 24 was higher in the combined infliximab groups, as well as the individual infliximab dose groups, compared with the placebo group ( $\mathrm{p}<0.01$ for all comparisons). The improvement in mean ePOST score was not maintained after infliximab treatment was discontinued.

After adjustment for the number of extrapulmonary organs involved, the improvement in the ePOSTadj score observed in the combined infliximab groups was also statistically significantly greater than that observed in placebo-treated patients at the week 18, 24, 30 and 44 evaluations ( $<<0.05$; fig. 3 ).

Table 6 describes the response of individual organs to infliximab compared with placebo. Due to the small sample sizes, no meaningful conclusions could be identified. Four organs were not reported in table 6 (throat, parotid/salivary glands, ear and gastrointestinal) because fewer than five subjects had involvement of these organs at both baseline and week 24. However in these four organs, the infliximab groups scored the same or better than the placebo group in every case. There were no substantial differences in the concomitant medications used for sarcoidosis treatment within

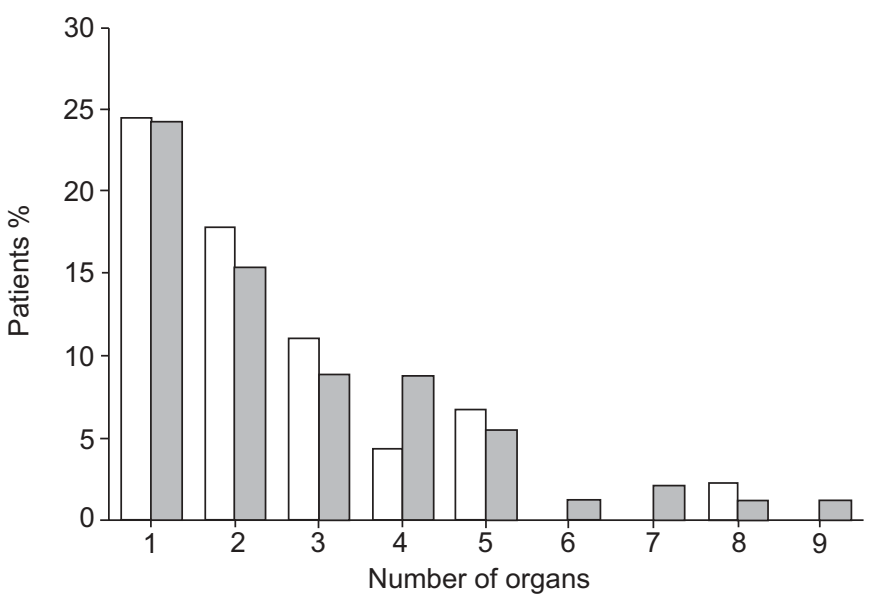

FIGURE 1. Bar graph of distribution of number of extrapulmonary organs involved, according to treatment group. $\square$ : placebo group; $\square$ : combined infliximab groups.

or between groups [21]. Therefore, a stratified analysis of ePOST scores was not required.

Table 7 shows the change in ePOST in two subgroups of organs, major and minor (defined previously in the Methods section), between week 0 and week 24. Compared with the placebo, infliximab improved the ePOST more in the major organs than in the minor organs, but not to a significant degree (for further details see online supplementary data).

\section{Adverse events}

The adverse events of the present study have been reported previously [21]. The proportions of patients who had adverse events were similar across the treatment groups. Infusion reactions occurred with $2.3 \%$ of infusions in both the placebo (six out of 258 infusions) and combined infliximab (12 out of 529 infusions) groups.

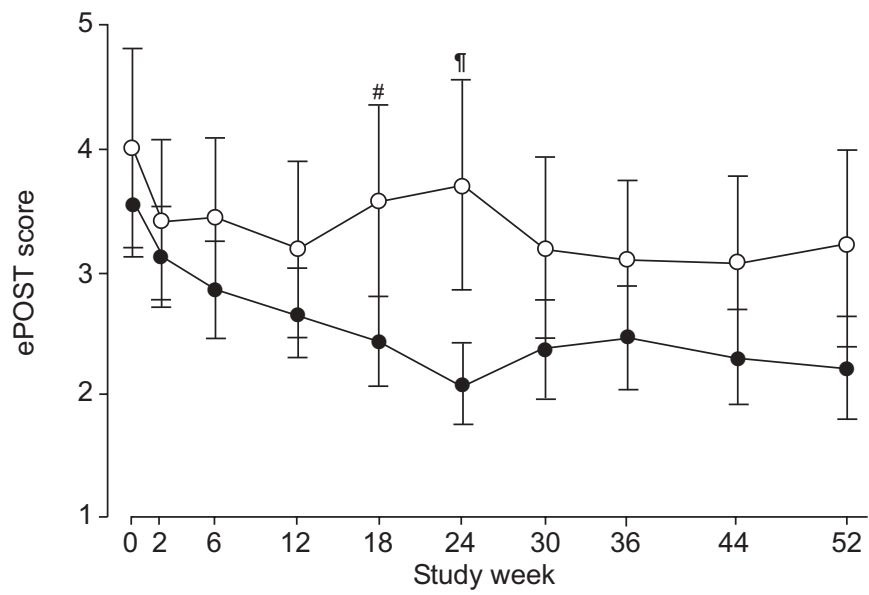

FIGURE 2. Mean extrapulmonary physician organ severity tool (ePOST) scores, with SEM bars, at each assessment visit, according to treatment group. $\bigcirc$ : placebo group; $\bullet$ : combined infliximab groups. ${ }^{*}: p=0.0247 ;{ }^{\bullet}: p=0.0019$. If not specified, $p>0.05$. Comparisons between groups are based upon change from baseline values. 


TABLE $5 \begin{aligned} & \text { Change in number of extrapulmonary organs } \\ & \text { involved from baseline to week } 24\end{aligned}$
$\begin{aligned} & \text { Placebo } \\ & \text { Combined infliximab }\end{aligned}$
$\begin{aligned} & \text { Total subjects } \\ & \text { Change in organ number } \\ & \leqslant-1\end{aligned}$
0

There were no anaphylactic or delayed hypersensitivity reactions reported during the study.

\section{DISCUSSION}

The present double-blind randomised study demonstrated that infliximab therapy improved extrapulmonary sarcoidosis compared with placebo, as assessed by a novel severity tool, the ePOST. Similar findings were observed when an adjustment was made for the number of extrapulmonary organs involved (ePOSTadj). All patients in the study were required to be receiving a stable dose of corticosteroid or another immunosuppressant to control their pulmonary sarcoidosis. Therefore, the results of the present trial suggest that infliximab provides additional benefit for extrapulmonary sarcoidosis beyond that achieved with standard sarcoidosis treatment.

The total score of extrapulmonary sarcoidosis severity was decreased by $>40 \%$ in the infliximab groups compared with placebo after 24 weeks of therapy. The difference between groups was not maintained after cessation of therapy at week 24 . This was primarily due to the worsening in the infliximab $3 \mathrm{mg} \cdot \mathrm{kg}^{-1}$ group [21]. Although investigators were encouraged to keep the dose of any concomitant medications as consistent as possible throughout the study, some patients did undergo modifications in their concomitant medication regimen. The impact of this remains unclear. In addition, the ePOST score also showed improvement in the infliximab groups compared with the placebo group when adjusted for the number of organs involved. Thus, the present authors believe that it was appropriate to calculate the ePOSTadj score as well as the ePOST score because, otherwise, small changes in a few organs were amplified in a severity score if a patient had a number of organs involved.

There are several potential limitations of this severity tool. First, the definition of involvement of sarcoidosis in an extrapulmonary organ was arbitrary, based on the clinical decision of the principal investigator at each clinical centre. However, the principal investigators in the present study were all experienced in the clinical presentation and management of sarcoidosis. Although data concerning the degree of certainty of the diagnosis of extrapulmonary organ involvement was not available, the diagnosis should be predominantly accurate, as three of the five most frequently identified extrapulmonary organs were the peripheral lymph nodes, skin and eyes (table 4). Confirmation of involvement of these organs was

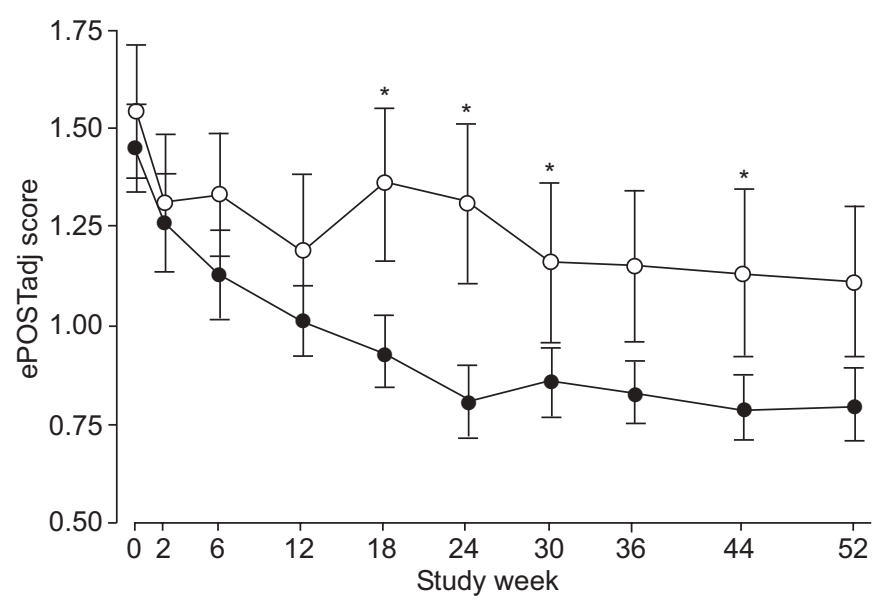

FIGURE 3. Mean adjusted extrapulmonary physician organ severity tool (ePOSTadj) scores, with SEM bars, at each assessment visit, according to treatment group. EPOSTadj is the ePOST score divided by the number of extrapulmonary organs involved at any time during the study. $\bigcirc$ : placebo group; $\mathbf{0}$ : combined infliximab groups. ${ }^{*}: p<0.05$.

probably by biopsy, the presence of lupus pernio facial skin lesions or by ophthalmological examination. These are very secure methods of diagnosis of sarcoidosis organ involvement in patients with biopsy-proven pulmonary sarcoidosis. However, detection of extrapulmonary organ involvement was not standardised. Therefore, changes in extrapulmonary organ involvement found by sensitive techniques (e.g. positron emission tomography scanning) may have escaped assessment in many patients. Secondly, the present severity tool has not been previously validated as reproducible or related to clinical outcome. Thirdly, the ePOST score was a summation of all extrapulmonary organ involvement and did not include weighting for specific organ involvement. Thus, major changes in one organ may have been diluted by the number of organs affected. For this reason, the ePOSTadj score was also calculated, in an attempt to adjust for this confounding effect. Also, patients with more extensive involvement of certain organ systems might have had more supplementary information available, upon which the physician could base their assessment. Additional measures to evaluate each organ system (i.e. laboratory analyses) were not mandated by the protocol and it is possible that some physicians may have had such information available to them while others did not.

The tool also weighted each organ similarly, while certain organs probably have a greater impact on clinical function and quality of life than others. For example, peripheral lymph nodes were the most common site of extrapulmonary sarcoidosis and, therefore, carried the most weight in the ePOST assessment system. It is very likely that other extrapulmonary organs were much more important. The subgroups of organs that have a potential major clinical impact ("major organs") and those that have a minor clinical impact ("minor organs") were, therefore, examined. Subgroup analyses, such as these, must be viewed with extreme caution, as the subgroups were formed arbitrarily and the sample sizes were smaller than for the entire cohort. Both subgroups demonstrated a reduction in ePOST between week 0 and 


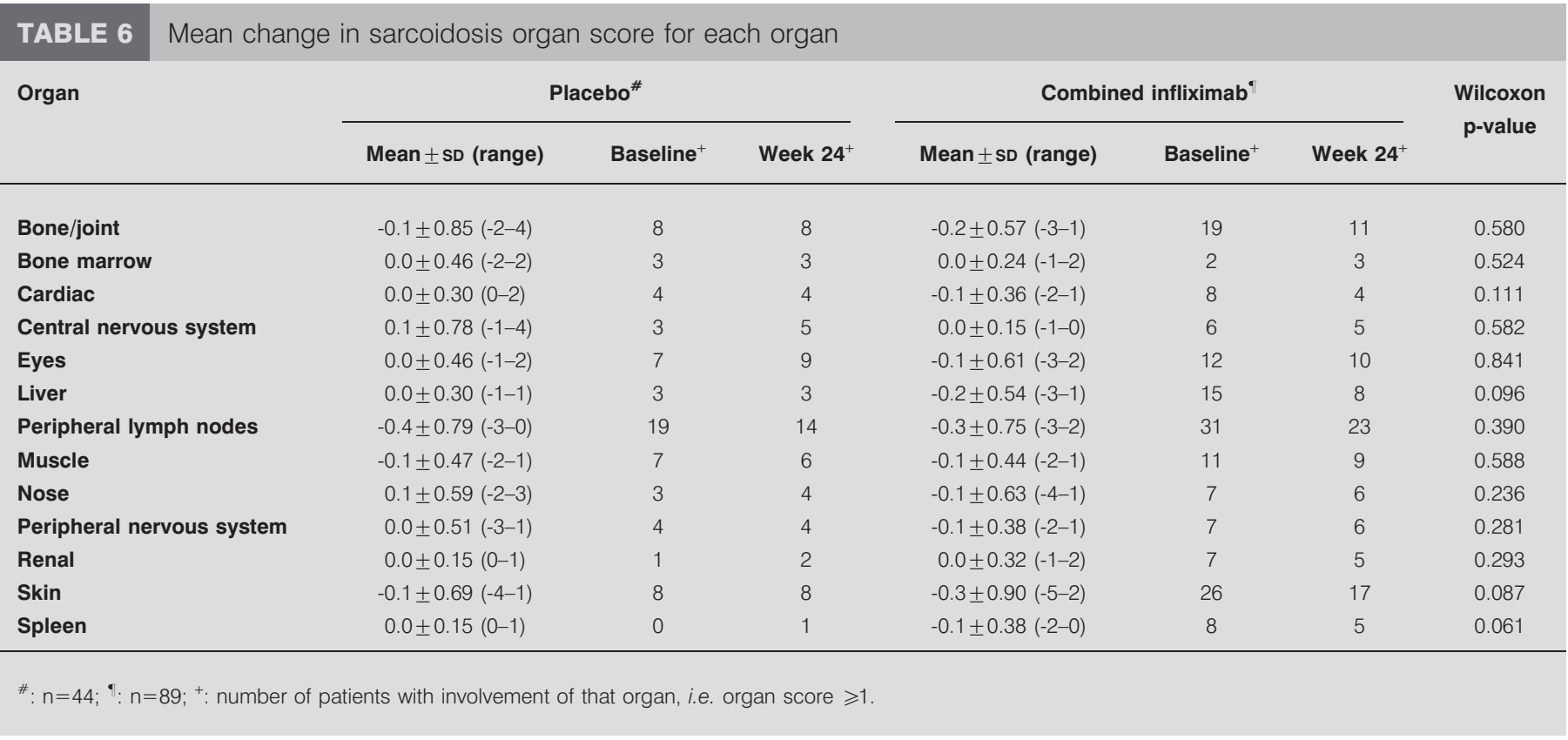

week 24. However, the ePOST did not reach statistical significance in either subgroup, most probably because of the smaller sample sizes when compared with the entire cohort. In addition, problems of reproducibility and subjectivity of the tool would be likely to create statistical noise and make the tool less reliable, which would tend to make the tool less likely to show differences between the placebo and infliximab groups. Nevertheless, statistical differences were noted between the placebo and infliximab groups despite these potential shortcomings. Another limitation was that the primary pulmonary study [21] and the present secondary extrapulmonary study may have suffered from a selection bias. Investigators may have opted to administer infliximab in an open-label fashion to patients with severe or progressive disease, thereby biasing enrolment in the present study towards subjects with milder disease. Such a bias may have made it more difficult to detect significant changes due to infliximab therapy, but this remains conjectural.

It should be noted that the severity of extrapulmonary sarcoidosis was a secondary end-point of the present study. Patients enrolled in this study were required to have the lung as the primary organ of sarcoidosis involvement and, although patients with extrapulmonary sarcoidosis were encouraged to participate, this was not an eligibility requirement. Thus, some patients with extrapulmonary manifestations of sarcoidosis had more organs involved than others. It is conceivable that patients with severe extrapulmonary sarcoidosis would have responded differently to infliximab. However, the fact that the present study patients with relatively mild extrapulmonary sarcoidosis demonstrated a response to infliximab suggests that this therapy might be effective for more severe extrapulmonary disease.

Another potential limitation of the present study is that the duration of therapy was only 24 weeks. There appears to be a dose-dependent increase in the risk of serious infections and malignancies with anti-TNF- $\alpha$ antibody therapy [23]. Therefore, the risk-benefit ratio of infliximab therapy for extrapulmonary sarcoidosis may not have been completely assessed in the present trial.

Other sarcoidosis instruments have been developed to assess the disease. A sarcoidosis assessment instrument was developed as part of the National Heart, Lung, and Blood Institute study "A Case-Control Etiologic Study of Sarcoidosis" (ACCESS) [24]. However, this instrument was not appropriate

\begin{tabular}{|c|c|c|c|c|c|c|c|}
\hline & \multicolumn{3}{|c|}{ Placebo $^{\#}$} & \multicolumn{3}{|c|}{ Combined infliximab } & \multirow{2}{*}{$\begin{array}{c}\text { Wilcoxon } \\
\text { p-value }\end{array}$} \\
\hline & Baseline & Week 24 & Change & Baseline & Week 24 & Change & \\
\hline ePOST minor & $2.50 \pm 3.91$ & $1.91 \pm 3.17$ & $-0.59 \pm 2.19$ & $2.28 \pm 2.96$ & $1.29 \pm 2.00$ & $-0.99 \pm 1.96$ & 0.174 \\
\hline
\end{tabular}

Data are presented as mean $\pm \mathrm{SD}$, unless otherwise stated. ePOST minor: sum of the scores for bone/joint, peripheral lymph nodes, muscle, nose, skin and spleen; ePOST major: sum of the scores for bone marrow, cardiac, central nervous system, peripheral nervous system, liver, renal and eyes. ${ }^{*}: n=44 ; ": n=89$. 
for the present study because it determines whether an organ is involved with sarcoidosis but does not assess the severity of organ involvement. Instead, the ACCESS instrument was used as a guide to define specific organ involvement. In addition, WASFI et al. [25] developed a sarcoidosis severity score that was derived by sarcoidosis experts who subjectively graded the severity of 100 vignettes of sarcoidosis cases. The vignettes were "broken down" into the items of objective information that they contained, and then a logistic regression analysis extracted the objective components upon which the experts scored disease severity. The resulting equation was then "validated" by three additional international sarcoidosis experts, who graded the same vignettes. Although this score developed by WASFI et al. [25] would have been of interest in the present study, it was published after the initiation of the present trial.

In conclusion, the results of the extrapulmonary physician organ severity tool assessment performed during the present randomised, double-blind, placebo-controlled study suggest that infliximab may be beneficial in the treatment of extrapulmonary sarcoidosis in patients already receiving corticosteroids. Further study of infliximab and other tumour necrosis factor- $\alpha$ antagonists is warranted in this group of patients.

\section{ACKNOWLEDGEMENTS}

The authors would like to acknowledge all of the investigators and patients who participated in this study. The Centocor T48 Sarcoidosis investigators from the 34 sites participating in the present study were as follows: R.P. Baughman (University of Cincinnati Medical Center, Cincinnati, OH); M.S. Kavuru and D.A. Culver (The Cleveland Clinic Foundation, Cleveland, $\mathrm{OH}$ ); G.S. Davis (University of Vermont Lung Center, Colchester, VT); C.M. Fogarty (Spartanburg Pharmaceutical Research, Spartanburg, SC); M.A. Judson (Medical University of South Carolina, Charleston, SC); G.W. Hunninghake (University of Iowa Hospitals and Clinics, Iowa City, IA); A.S. Teirstein (Mount Sinai Medical Center, New York, NY); M. Mandel (Pulmonary Research Associates, LLC, Larchmont, NY); D. McNally (University of Connecticut Health Center, Farmington, CT); L. Tanoue (Yale University School of Medicine, New Haven, CT); L. Newman and Y. Wasfi (National Jewish Medical and Research Center, Denver, CO); H. Patrick (Drexel University College of Medicine, Philadelphia, PA); M.D. Rossman (Hospital of the University of Pennsylvania, Philadelphia, PA); G. Raghu (University of Washington Medical Center, Seattle, WA); O. Sharma (University of Southern California, Los Angeles, CA); D. Wilkes (Indiana University, Indianapolis, IN); H. Yeager (Georgetown University Medical Center, Washington, DC); J.F. Donahue (University of North Carolina at Chapel Hill, Chapel Hill, NC); M. Kaye (Minnesota Lung Center, Minneapolis, MN); N. Sweiss (University of Chicago, Chicago, IL; all USA); N. Vetter (Otto Wagner Hospital, Pulmonologische Zentrum, Vienna, Austria); M. Thomeer (Universitair Ziekenhuis Leuven, Campus Gasthuisberg, Leuven, Belgium); M. Brutsche (University Hospital Basel, Basel, Switzerland); L. Nicod (Inselspital Bern, Bern, Switzerland); D. Valeyre (Hopital Avicenne, Bobigny, France); P. Chanez (Clinique des Maladies Respiratoires,
Hopital Arnaud de Villeneuve, Montpellier, France); C. Albera (Ospedale San Luigi, Orbassano, Italy); M. Drent (University Hospital Maastricht, Maastricht, the Netherlands); J. Grutters (St Antonius Hospital, Heart Lung Center Utrecht, Nieuwegein, the Netherlands); H. Hoogsteden (Erasmus MC Rotterdam, Rotterdam, the Netherlands); U. Costabel (Ruhrlandklinik, Essen, Germany); J. MullerQuernheim (Universitätsklinik Freiburg, Freiburg, Germany); R. Bonnet (Klinik für Pneumologie, Zentralklinik Bad Berka, Bad Berka, Germany); and F. Kanniess (Pulmonary Research Institute, Hospital Grosshansdorf, Grosshansdorf, Germany).

\section{REFERENCES}

1 Judson MA. An approach to the treatment of pulmonary sarcoidosis with corticosteroids: the six phases of treatment. Chest 1999; 115: 1158-1165.

2 Lynch JP 3rd, Kazerooni EA, Gay SE. Pulmonary sarcoidosis. Clin Chest Med 1997; 18: 755-785.

3 Baughman RP, Lower EE. A clinical approach to the use of methotrexate for sarcoidosis. Thorax 1999; 54: 742-746.

4 Jones E, Callen JP. Hydroxychloroquine is effective therapy for control of cutaneous sarcoidal granulomas. $J$ Am Acad Dermatol 1990; 23: 487-489.

5 Müller-Quernheim J, Kienast K, Held M, Pfeifer S, Costabel U. Treatment of chronic sarcoidosis with an azathioprine/prednisolone regimen. Eur Respir J 1999; 14: 1117-1122.

6 Lower EE, Broderick JP, Brott TG, Baughman RP. Diagnosis and management of neurological sarcoidosis. Arch Intern Med 1997; 157: 1864-1868.

7 Doty JD, Mazur JE, Judson MA. Treatment of corticosteroid-resistant neurosarcoidosis with a shortcourse cyclophosphamide regimen. Chest 2003; 124: 2023-2026.

8 Judson MA, Silvestri J, Hartung C, Byars T, Cox CE. The effect of thalidomide on corticosteroid-dependent pulmonary sarcoidosis. Sarcoidosis Vasc Diffuse Lung Dis 2006; 23: 51-57.

9 Zabel P, Entzian P, Dalhoff K, Schlaak M. Pentoxifylline in treatment of sarcoidosis. Am J Respir Crit Care Med 1997; 155: 1665-1669.

10 Baughman RP, Lower EE. Infliximab for refractory sarcoidosis. Sarcoidosis Vasc Diffuse Lung Dis 2001; 18: 70-74.

11 Doty JD, Mazur JE, Judson MA. Treatment of sarcoidosis with infliximab. Chest 2005; 127: 1064-1071.

12 Heffernan MP, Anadkat MJ. Recalcitrant cutaneous sarcoidosis responding to infliximab. Arch Dermatol 2005; 141: 910-911.

13 Carter JD, Valeriano J, Vasey FB, Bognar B. Refractory neurosarcoidosis: a dramatic response to infliximab. Am J Med 2004; 15, 117: 277-279.

14 Hoitsma E, Faber CG, van Santen-Hoeufft M, De Vries J, Reulen JP, Drent M. Improvement of small fiber neuropathy in a sarcoidosis patient after treatment with infliximab. Sarcoidosis Vasc Diffuse Lung Dis 2006; 23: 73-77.

15 Fehrenbach H, Zissel G, Goldmann T, et al. Alveolar macrophages are the main source for tumour necrosis factor- $\alpha$ in patients with sarcoidosis. Eur Respir J 2003; 21: $421-428$ 
16 Zissel G, Müller-Quernheim J. Sarcoidosis: historical perspective and immunopathogenesis (Part I). Respir Med 1998; 92: 126-139.

17 Ziegenhagen MW, Rothe ME, Zissel G, MüllerQuernheim J. Exaggerated $\mathrm{TNF} \alpha$ release of alveolar macrophages in corticosteroid resistant sarcoidosis. Sarcoidosis Vasc Diffuse Lung Dis 2002; 19: 185-190.

18 Mañá J, Marcoval J, Graells J, Salazar A, Peyrí J, Pujol R. Cutaneous involvement in sarcoidosis. Relationship to systemic disease. Arch Dermatol 1997; 133: 882-888.

19 Sharma OP. Sarcoidosis of the upper respiratory tract. Selected cases emphasizing diagnostic and therapeutic difficulties. Sarcoidosis Vasc Diffuse Lung Dis 2002; 19: 227-233.

20 Scallon B, Cai A, Solowski N, et al. Binding and functional comparisons of two types of tumor necrosis factor antagonists. J Pharmacol Exp Ther 2002; 301: 418-426.

21 Baughman RP, Drent M, Kavuru M, et al. Infliximab therapy in patients with chronic sarcoidosis and pulmonary involvement. Am J Respir Crit Care Med 2006; 174: 795-802.

22 Fletcher CM. Standardised questionnaire on respiratory symptoms: a statement prepared and approved by the MRC Committee on the Aetiology of Chronic Bronchitis (MRC breathlessness score). BMJ 1960; 2: 1665.

23 Bongartz T, Sutton AJ, Sweeting MJ, Buchan I, Matteson EL, Montori V. Anti-TNF antibody therapy in rheumatoid arthritis and the risk of serious infections and malignancies: systemic review and meta-analysis of rare harmful effects in randomized controlled trials. JAMA 2006; 295: 2275-2285.

24 Judson MA, Baughman RP, Teirstein AS, Terrin ML, Yeager H Jr. Defining organ involvement in sarcoidosis: the ACCESS proposed instrument. ACCESS Research Group. A Case-Control Etiologic Study of Sarcoidosis. Sarcoidosis Vasc Diffuse Lung Dis 1999; 16: 75-86.

25 Wasfi YS, Rose CS, Murphy JR, et al. A new tool to assess sarcoidosis severity. Chest 2006; 129: 1234-1245. 